Research Article

\title{
Fixed Point of Orthogonal F-Suzuki Contraction Mapping on O-Complete b-Metric Spaces with Applications
}

\author{
Ismat Beg $\mathbb{D}^{1},{ }^{1}$ Gunaseelan Mani $\mathbb{D}^{2},{ }^{2}$ and Arul Joseph Gnanaprakasam ${ }^{3}$ \\ ${ }^{1}$ Centre for Mathematics and Statistical Sciences, Lahore School of Economics, Lahore 53200, Pakistan \\ ${ }^{2}$ Department of Mathematics, Sri Sankara Arts and Science College (Autonomous), Affiliated to Madras University, Enathur, \\ Kanchipuram, Tamil Nadu 631 561, India \\ ${ }^{3}$ Department of Mathematics, College of Engineering and Technology, Faculty of Engineering and Technology, SRM Institute of \\ Science and Technology, SRM Nagar, Kattankulathur 603203, Kanchipuram, Chennai, Tamil Nadu, India \\ Correspondence should be addressed to Ismat Beg; ibeg@lahoreschool.edu.pk
}

Received 2 December 2020; Revised 19 February 2021; Accepted 2 March 2021; Published 10 March 2021

Academic Editor: Calogero Vetro

Copyright (c) 2021 Ismat Beg et al. This is an open access article distributed under the Creative Commons Attribution License, which permits unrestricted use, distribution, and reproduction in any medium, provided the original work is properly cited.

In this paper, we introduce the concept of generalized orthogonal $F$-Suzuki contraction mapping and prove some fixed point theorems on orthogonal $b$-metric spaces. Our results generalize and extend some of the well-known results in the existing literature. As an application of our results, we show the existence of a unique solution of the first-order ordinary differential equation.

\section{Introduction and Preliminaries}

Banach contraction principle is one of the famous and useful results in mathematics. In last 100 years, it is extended in many different directions. The substitution of the metric space by other generalized metric spaces is one normal way to strengthen the Banach contraction principle. The concept of a $b$-metric space was introduced by Bakhtin [1] and Czerwik [2]. They also established the fixed point result in the setting of $b$-metric spaces which is a generalization of the Banach contraction principle. In 2015, Alsulami et al. [3] introduced the concepts of generalized F-Suzuki type contraction mappings and proved the fixed point theorems on complete $b$-metric spaces. On the other hand, Gordji et al. [4] introduced the new concept of an orthogonality in metric spaces and proved the fixed point result for contraction mappings in metric spaces endowed with this new type of orthogonality. Furthermore, they gave the application of this results for the existence and uniqueness of the solution of a first-order ordinary differential equation, while the Banach contraction mapping principle cannot be applied in this situation. This new concept of an orthogonal set has many applications, and there are also many types of the orthogonality. Afterward Eshaghi Gordji and Habibi [5] proved fixed point in generalized orthogonal metric spaces. Recently, Sawangsup et al. [6] introduced the new concept of an orthogonal $F$-contraction mappings and proved the fixed point theorems on orthogonal-complete metric spaces. Subsequently, many other researchers [7-11] studied the orthogonal contractive type mappings and obtained significant results. This paper is in continuation of these studies; first, we introduced the new concepts of generalized orthogonal $F$-Suzuki contraction mappings on an orthogonal $b$ metric space and then prove the fixed point theorems on orthogonal $b$-complete metric space with examples and applications to differential equations. Over results generalize/extend several results from the existing literature.

In this paper, we denote by $\mathbb{N}, \mathbb{R}^{+}$, and $W$ the set of positive integers, the set of positive real numbers, and the nonempty set, respectively. Next, we state the concept of a control function which was introduced by Wardowski [12].

Let $\mathfrak{I}$ denote the family of all functions $F: \mathbb{R}^{+} \longrightarrow \mathbb{R}$ satisfying the following properties:

$\left(\mathrm{F}_{1}\right) F$ is strictly increasing

$\left(\mathrm{F}_{2}\right)$ for each sequence $\left\{\alpha_{n}\right\}$ of positive numbers, we have

$$
\lim _{n \rightarrow \infty} \alpha_{n}=0 \Leftarrow \lim _{n \rightarrow \infty} F\left(\alpha_{n}\right)=-\infty
$$


Bakhtin [1] and Czerwik [2] gave the concept of a $b$ metric space as follows.

Definition 1 (see [2]). Let $W$ be a nonempty set and $s \geq 1$. Suppose that the mapping $d: W \times W \longrightarrow \mathbb{R}^{+}$satisfies the following conditions for all $u, v, w \in W$ :

(i) $d(u, v)=0$ if and only if $u=v$

(ii) $d(u, v)=d(v, u)$

(iii) $d(u, v) \leq s[d(u, w)+d(w, v)]$

Then, $(W, d)$ is called a $b$-metric space with the coefficient $s$.

Example 2 (see [2]). Define a mapping $d: W \times W \longrightarrow \mathbb{R}^{+}$by $d(u, v)=|u-v|^{2}$ for all $u, v \in \mathbb{W}$. Then, $(W, d)$ is a $b$-metric space with the coefficient $s=2$.

The idea of generalized $F$-Suzuki type contraction and $F$ Suzuki type contraction in complete $b$-metric spaces is due to Alsulami et al. [3]. Gordji et al. [4] introduced the notion of an orthogonal set (or $O$-set).

Definition 3 (see [4]). Let $W \neq \phi$ and $\perp \subseteq W \times W$ be a binary relation. If $\perp$ satisfies the following condition:

$$
\exists u_{0} \in W:\left(\forall u \in W, u \perp u_{0}\right) \text { or }\left(\forall u \in W, u_{0} \perp u\right)
$$

then it is called an orthogonal set (briefly $O$-set). We denote this $O$-set by $(W, \perp)$.

Example 4 (see [4]). Let $W$ be the set all people in the world. Define the binary relation $\perp$ on $W$ by $v \perp u$ if $v$ can give blood to $u$. According to the Table 1 , if $u_{0}$ is a person such that his (her) blood type is $O$-, then we have $u_{0} \perp u$ for all $u \in W$. This means that $(W, \perp)$ is an $O$-set. In this $O$-set, $u_{0}$ (in Definition 3 ) is not unique. Note that, in this example, $u_{0}$ may be a person with blood type $A B+$. In this case, we have $u \perp u_{0}$ for all $u \in W$.

Next, we give some basic definition for subsequent use.

Definition 5 (see [4]). Let $(W, \perp)$ be an $O$-set. A sequence $\left\{u_{n}\right\}$ is called an orthogonal sequence (briefly, $O$-sequence) if

$$
\left(\forall n \in \mathbb{N}, u_{n} \perp u_{n+1}\right) \text { or }\left(\forall n \in \mathbb{N}, u_{n+1} \perp u_{n}\right) .
$$

Definition 6 (see [4]). The triplet $(W, \perp, d)$ is called an orthogonal metric space if $(W, \perp)$ is an $O$-set and $(W, d)$ is a metric space.

Definition 7 (see [4]). Let $(W, \perp, d)$ be an orthogonal metric space. Then, a mapping $G: W \longrightarrow W$ is said to be orthogonally continuous (or $\perp$-continuous) in $u \in W$ if for each $O$-sequence $\left\{u_{n}\right\}$ in $W$ with $u_{n} \longrightarrow u$ as $n \longrightarrow \infty$, we have $G\left(u_{n}\right) \longrightarrow G(u)$ as $n \longrightarrow \infty$. Also, $G$ is said to be $\perp$-continuous on $W$ if $G$ is $\perp$-continuous in each $u \in W$.
TABLE 1: Blood types.

\begin{tabular}{lcc}
\hline Type & You can give blood to & You can receive blood from \\
\hline $\mathrm{A}+$ & $\mathrm{A}+\mathrm{AB}+$ & $\mathrm{A}+\mathrm{A}-\mathrm{O}+\mathrm{O}-$ \\
$\mathrm{O}+$ & $\mathrm{O}-\mathrm{A}+\mathrm{B}+\mathrm{AB}+$ & $\mathrm{O}+\mathrm{O}-$ \\
$\mathrm{B}+$ & $\mathrm{B}+\mathrm{AB}+$ & $\mathrm{B}+\mathrm{B}-\mathrm{O}+\mathrm{O}-$ \\
$\mathrm{AB}+$ & $\mathrm{AB}+$ & Everyone \\
$\mathrm{A}-$ & $\mathrm{A}+\mathrm{A}-\mathrm{AB}+\mathrm{AB}-$ & $\mathrm{A}-\mathrm{O}-$ \\
$\mathrm{O}-$ & Everyone & $\mathrm{O}-$ \\
$\mathrm{B}-$ & $\mathrm{B}+\mathrm{B}-\mathrm{AB}+\mathrm{AB}-$ & $\mathrm{B}-\mathrm{O}-$ \\
$\mathrm{AB}-$ & $\mathrm{AB}+\mathrm{AB}-$ & $\mathrm{AB}-\mathrm{B}-\mathrm{O}-\mathrm{A}-$ \\
\hline
\end{tabular}

Remark 8 (see [4]). Every continuous mapping is $\perp$-continuous and the converse is not true.

Definition 9 (see [4]). Let $(W, \perp, d)$ be an orthogonal metric space. Then, $W$ is said to be orthogonally complete (briefly, $\mathrm{O}$-complete) if every Cauchy $\mathrm{O}$-sequence is convergent.

Remark 10 (see [4]). Every complete metric space is $\mathrm{O}$ complete and the converse is not true.

Definition 11 (see [4]). Let $(W, \perp)$ be an $O$-set. A mapping $G: W \longrightarrow W$ is said to be $\perp$-preserving if $G u \perp G v$ when ever $u \perp v$.

An orthogonal sequence $\left\{u_{n}\right\}$ is said to satisfy the condition $(T)$ if

$$
\left(\forall n, m \in \mathbb{N}, u_{n} \perp u_{m}\right) \text { or }\left(\forall n, m \in \mathbb{N}, u_{m} \perp u_{n}\right) .
$$

Throughout the paper, we assume that an orthogonal sequence $\left\{u_{n}\right\}$ satisfies the condition $(T)$.

\section{Main Results}

In this section, inspired by Alsulami et al. [3], the notion of a generalized $F$-Suzuki type contraction mapping, we introduce a new generalized $F$-Suzuki contraction mapping and obtain fixed point results for this contraction mapping on an orthogonal $b$-metric space.

Definition 12. Let $(W, \perp, d)$ be an orthogonal b-metric space with constant $s \geq 1$. A map $G: W \longrightarrow W$ is said to be a generalized orthogonal $F$-Suzuki contraction mapping (briefly, generalized $G_{\perp}$-Suzuki contraction) on $(W, \perp, d)$ if there are $F \in \mathfrak{I}$ and $\tau>0$ such that the following condition holds:

$$
\begin{aligned}
\forall u, v \in & W \text { with } u \perp v\left[d(G u, G v)>0, \frac{1}{2 s} d(u, G u)<d(u, v)\right. \\
\Longrightarrow & \tau+F(d(G u, G v)) \leq a F(d(u, v))+b F(d(u, G u)) \\
& +c F(d(v, G v))],
\end{aligned}
$$

where $c \in[0,1)$ and $a, b \in[0,1]$ are real numbers with $a+$ $b+c=1$. 
In the above Definition 12, if we take $a=1$ and $b=c=0$; we obtain the following Definition.

Definition 13. Let $(W, \perp, d)$ be an orthogonal $b$-metric space with constant $s \geq 1$. A map $G: W \longrightarrow W$ is said to be an orthogonal F -Suzuki contraction mapping (briefly, $G_{\perp}$ Suzuki contraction) on $(W, \perp, d)$ if there are $F \in \mathfrak{I}$ and $\tau>0$ such that the following condition holds:

$$
\begin{aligned}
& \forall u, v \in W \text { with } u \perp v\left[d(G u, G v)>0, \frac{1}{2 s} d(u, G u)<d(u, v)\right. \\
&\Longrightarrow \tau+F(d(G u, G v)) \leq F(d(u, v))] .
\end{aligned}
$$

Theorem 14. Let $(W, \perp, d)$ be an $O$-complete orthogonal $b$ metric space with constant $s \geq 1$ and an orthogonal element $u_{0}$ and a mapping $G: W \longrightarrow W$. Suppose that there exist $F \in \mathfrak{I}$ and $\tau>0$ such that the following conditions hold:

(i) $G$ is $\perp$-preserving

(ii) $G$ is generalized $G_{\perp}$-Suzuki contraction mapping

Then, $G$ has a fixed point $t \in W$, and for every $u \in W$, the sequence $\left\{G^{n} u\right\}$ converges to $t$.

Proof. Since $(W, \perp)$ is an $O$-set,

$$
\exists u_{0} \in W:\left(\forall u \in W, u \perp u_{0}\right) \text { or }\left(\forall u \in W, u_{0} \perp u\right) .
$$

It follows that $u_{0} \perp G u_{0}$ or $G u_{0} \perp u_{0}$. Let

$$
u_{1}:=G u_{0}, u_{2}:=G u_{1}=G^{2} u_{0} \cdots \cdots, u_{n+1}:=G u_{n}=G^{n+1} u_{0},
$$

for all $n \in \mathbb{N} \cup\{0\}$. If $u_{n}=u_{n+1}$ for any $n \in \mathbb{N} \cup\{0\}$, then it is clear that $u_{n}$ is a fixed point of $G$. Assume that $u_{n} \neq u_{n+1}$ for all $n \in \mathbb{N} \cup\{0\}$. Thus, we have $d\left(G u_{n}, G u_{n+1}\right)>0$ for all $n \in \mathbb{N} \cup\{0\}$. Since $G$ is $\perp$-preserving, we have

$$
u_{n} \perp u_{n+1} \text { or } u_{n+1} \perp u_{n}
$$

for all $n \in \mathbb{N} \cup\{0\}$. This implies that $\left\{u_{n}\right\}$ is an $O$-sequence. Since $G$ is generalized $G_{\perp}$-Suzuki contraction mapping, we have

$$
\left.\frac{1}{2 s} d\left(u_{n}, G u_{n}\right)\right)<d\left(u_{n}, G u_{n}\right), \forall n \in \mathbb{N}
$$

Thus, by hypothesis of theorem, we have

$$
\begin{aligned}
\tau+F\left(d\left(G u_{n}, G^{2} u_{n}\right)\right) \leq & a F\left(d\left(u_{n}, G u_{n}\right)\right)+b F\left(d\left(u_{n}, G u_{n}\right)\right) \\
& +c F\left(d\left(G u_{n}, G^{2} u_{n}\right)\right),
\end{aligned}
$$

which is equivalent to

$$
\tau+(1-c) F\left(d\left(G u_{n}, G^{2} u_{n}\right)\right) \leq(a+b) F\left(d\left(u_{n}, G u_{n}\right)\right) .
$$

Since $a+b+c=1$, the inequality (12) turns into

$$
F\left(d\left(G u_{n}, G^{2} u_{n}\right)\right) \leq F\left(d\left(u_{n}, G u_{n}\right)\right)-\frac{\tau}{a+b}<F\left(d\left(u_{n}, G u_{n}\right)\right) .
$$

From $\left(F_{1}\right)$, we conclude that

$$
d\left(u_{n+1}, G u_{n+1}\right)=d\left(G u_{n}, G^{2} u_{n}\right)<d\left(u_{n}, G u_{n}\right), \forall n \in \mathbb{N} .
$$

Therefore, $\left\{d\left(u_{n}, G u_{n}\right)\right\}_{n=1}^{\infty}$ is a decreasing sequence of real numbers which is bounded below. Therefore, $\left\{d\left(u_{n}, G u_{n}\right)\right\}_{n=1}^{\infty}$ converges and

$$
\lim _{n \rightarrow \infty} d\left(u_{n}, G u_{n}\right)=\delta=\inf \left\{d\left(u_{n}, G u_{n}\right): \forall n \in \mathbb{N}\right\} .
$$

We will show that $\delta=0$. On the contrary, suppose that $\delta$ $>0$. In other words, for every $\varepsilon>0$ there exits $k \in \mathbb{N}$, such that

$$
d\left(u_{k}, G u_{k}\right)<\delta+\varepsilon .
$$

From $\left(F_{1}\right)$, we find that

$$
F\left(d\left(u_{k}, G u_{k}\right)\right)<F(\delta+\varepsilon) .
$$

On the other hand, we have

$$
\left.\frac{1}{2 s} d\left(u_{k}, G u_{k}\right)\right)<d\left(u_{k}, G u_{k}\right),
$$

due to (10). Since $G$ is generalized $G_{\perp}$-Suzuki contraction, we derive

$$
\begin{aligned}
\tau+F\left(d\left(G u_{k}, G^{2} u_{k}\right)\right) \leq & a F\left(d\left(u_{k}, G u_{k}\right)\right)+b F\left(d\left(u_{k}, G u_{k}\right)\right) \\
& +c F\left(d\left(G u_{k}, G^{2} u_{k}\right)\right)
\end{aligned}
$$

which yields that

$$
\tau+(1-c) F\left(d\left(G u_{k}, G^{2} u_{k}\right)\right) \leq(a+b) F\left(d\left(u_{k}, G u_{k}\right)\right),
$$

taking the fact that $a+b+c=1$ into account, we obtain

$$
F\left(d\left(G u_{k}, G^{2} u_{k}\right)\right) \leq F\left(d\left(u_{k}, G u_{k}\right)\right)-\frac{\tau}{a+b} .
$$

Analogously, again by (10), we have $(1 / 2 s) d\left(G u_{k}\right.$, $\left.\left.G^{2} u_{k}\right)\right)<d\left(G u_{k}, G^{2} u_{k}\right)$. Owing to the fact that $G$ is generalized $G_{\perp}$-Suzuki contraction, we conclude that

$$
\begin{aligned}
\tau+F\left(d\left(G^{2} u_{k}, G^{3} u_{k}\right)\right) \leq & a F\left(d\left(G u_{k}, G^{2} u_{k}\right)\right)+b F\left(d\left(G u_{k}, G^{2} u_{k}\right)\right) \\
& +c F\left(d\left(G^{2} u_{k}, G^{3} u_{k}\right)\right) .
\end{aligned}
$$


It implies that

$$
F\left(d\left(G^{2} u_{k}, G^{3} u_{k}\right)\right) \leq F\left(d\left(G u_{k}, G^{2} u_{k}\right)\right)-\frac{\tau}{a+b},
$$

since $a+b+c=1$. Furthermore, by combining (21) and (23), we get

$$
\begin{aligned}
F\left(d\left(G^{2} u_{k}, G^{3} u_{k}\right)\right) & \leq F\left(d\left(G u_{k}, G^{2} u_{k}\right)\right)-\frac{\tau}{a+b} \\
& \leq F\left(d\left(u_{k}, G u_{k}\right)\right)-\frac{2 \tau}{a+b} .
\end{aligned}
$$

Iteratively, we obtain that

$$
\begin{aligned}
F\left(d\left(G^{n} u_{k}, G^{n+1} u_{k}\right)\right) & \leq F\left(d\left(G^{n} u_{k}, G^{n-1} u_{k}\right)\right)-\frac{\tau}{a+b} \\
& \leq F\left(d\left(G^{n-1} u_{k}, G^{n-2} u_{k}\right)\right)-\frac{2 \tau}{a+b} \vdots \\
& \leq F\left(d\left(G u_{k}, u_{k}\right)\right)-\frac{n \tau}{a+b} \\
& \leq F(\delta+\varepsilon)-\frac{n \tau}{a+b} .
\end{aligned}
$$

By letting $n \longrightarrow \infty$, we find that

$$
\lim _{n \rightarrow \infty} F\left(d\left(G^{n} u_{k}, G^{n+1} u_{k}\right)\right)=-\infty
$$

Consequently, from $\left(F_{2}\right)$, we derive that $\lim _{n \rightarrow \infty} d\left(G^{n} u_{k}\right.$, $\left.G^{n+1} u_{k}\right)=0$. Thus, there exists $N_{1} \in \mathbb{N}$ such that

$$
d\left(G^{n} u_{k}, G^{n+1} u_{k}\right)<\delta, \forall n \geq N_{1}
$$

and from (8), we get

$$
d\left(u_{k+n}, G u_{k+n}\right)<\delta, \forall n \geq N_{1} .
$$

This is a contradiction with the definition of $\delta$. Hence, we have

$$
\lim _{n \rightarrow \infty} d\left(u_{n}, G u_{n}\right)=0
$$

It what follows, we will prove that

$$
\lim _{n, k \rightarrow \infty} d\left(u_{n}, u_{k}\right)=0
$$

Suppose, on the contrary, that there exists $\varepsilon>0$ and sequences $\{i(n)\}_{n=1}^{\infty}$ and $\{j(n)\}_{n=1}^{\infty}$ of natural numbers such that

$i(n)>j(n)>n, d\left(u_{i(n)}, u_{j(n)}\right) \geq \varepsilon, d\left(u_{i(n)-1}, u_{j(n)}\right)<\varepsilon, \forall n \in \mathbb{N}$.
From the triangle inequality, we have

$$
\begin{aligned}
d\left(u_{i(n)}, u_{j(n)}\right) & \leq s\left[d\left(u_{i(n)}, u_{i(n)-1}\right)+d\left(u_{i(n)-1}, u_{j(n)}\right)\right] \\
& \leq s d\left(u_{i(n)}, u_{i(n)-1}\right)+s \varepsilon \\
& =s d\left(u_{i(n)-1}, G u_{i(n)-1}\right)+s \varepsilon, \quad \forall n \in \mathbb{N}
\end{aligned}
$$

Owing to (29), there exists and $N_{2} \in \mathbb{N}$ such that

$$
d\left(u_{i(n)}, G u_{i(n)}\right)<\varepsilon, \forall n>N_{2}
$$

Taking (33) into account, (32) yields that

$$
d\left(u_{i(n)}, u_{j(n)}\right)<2 s \varepsilon, \forall n>N_{2} .
$$

So from $\left(F_{2}\right)$, we obtain

$$
F\left(d\left(u_{i(n)}, u_{j(n)}\right)\right)<F(2 s \varepsilon), \forall n>N_{2}
$$

On the other hand, we can easily get that

$\frac{1}{2 s} d\left(u_{i(n)}, G u_{i(n)}\right)<\frac{\varepsilon}{2 s}<\varepsilon \leq d\left(u_{i(n)}, u_{j(n)}\right), \forall n>N_{2}$.

Next, we claim that

$d\left(G u_{i(n)}, G u_{j(n)}\right)=d\left(u_{i(n)+1}, u_{j(n)+1}\right)>0, \forall n \in \mathbb{N}$.

Arguing by contradiction, there exists $k \geq \mathbb{N}$ such that

$$
d\left(u_{i(k)+1}, u_{j(k)+1}\right)=0
$$

It follows from (31), (36), and (38) that

$$
\begin{aligned}
\varepsilon & \leq d\left(u_{i(k)}, u_{j(k)}\right) \leq d\left(u_{i(k)}, u_{i(k)+1}\right)+d\left(u_{i(k)+1}, u_{j(k)}\right) \\
& \leq d\left(u_{i(k)}, u_{i(k)+1}\right)+d\left(u_{i(k)+1}, u_{j(k)+1}\right)+d\left(u_{j(k)+1}, u_{j(k)}\right) \\
& =d\left(u_{i(k)}, G u_{i(k)}\right)+d\left(u_{i(k)+1}, u_{j(k)+1}\right)+d\left(u_{j(k)}, G u_{j(k)}\right) \\
& <\frac{\varepsilon}{2 s}+0+\frac{\varepsilon}{2 s}=\frac{\varepsilon}{s} .
\end{aligned}
$$

This contradiction establishes the relation (37). Since $G$ is $\perp$-preserving, we have

$$
u_{i(n)} \perp u_{j(n)} \text { or } u_{j(n)} \perp u_{i(n)} .
$$


Since $G$ is generalized $G_{\perp}$-Suzuki contraction, $\forall n>N_{2}$, we find that

$$
\begin{aligned}
\tau+ & F\left(d\left(G u_{i(n)}, G u_{j(n)}\right)\right) \\
\leq & a F\left(d\left(u_{i(n)}, u_{j(n)}\right)\right)+b F\left(d\left(u_{i(n)}, G u_{i(n)}\right)\right) \\
& +c F\left(d\left(u_{j(n)}, G u_{j(n)}\right)\right) .
\end{aligned}
$$

Taking (35) into account, (41) turns into

$$
\begin{aligned}
\tau+F\left(d\left(G u_{i(n)}, G u_{j(n)}\right)\right)< & a F(2 s \varepsilon)+b F\left(d\left(u_{i(n)}, G u_{i(n)}\right)\right) \\
& +c F\left(d\left(u_{j(n)}, G u_{j(n)}\right)\right), \forall n>N_{2} .
\end{aligned}
$$

Regarding (29) and $\left(F_{2}\right)$, we obtain that

$$
\lim _{n \rightarrow \infty} F\left(d\left(G u_{i(n)}, G u_{j(n)}\right)\right)=-\infty
$$

From $\left(F_{2}\right)$, we get that

$\lim _{n \rightarrow \infty} F\left(d\left(G u_{i(n)}, G u_{j(n)}\right)\right)=0 \Leftrightarrow \lim _{n \rightarrow \infty} F\left(d\left(u_{i(n)+1}, u_{j(n)+1}\right)\right)=0$.

This is a contradiction with relations in (31). Hence, $\lim _{k, n \rightarrow \infty} d\left(u_{n}, u_{k}\right)=0$; that is $\left\{u_{n}\right\}_{n=1}^{\infty}$ is a Cauchy sequence in $W$. On the account of completeness of $(W, d)$, there exists $t \in W$ such that

$$
\lim _{n \rightarrow \infty} d\left(u_{n}, t\right)=0
$$

We claim that, for every $n \in \mathbb{N}$,

$\frac{1}{2 s} d\left(u_{n}, G u_{n}\right)<d\left(u_{n}, t\right)$ or $\frac{1}{2 s} d\left(G u_{n}, G^{2} u_{n}\right)<d\left(G u_{n}, t\right), \forall n \in \mathbb{N}$.

We will prove the claim above by the method of reductio ad absurdum. Suppose, on the contrary, that there exists $k \in \mathbb{N}$ such that

$$
\begin{gathered}
\frac{1}{2 s} d\left(u_{k}, G u_{k}\right) \geq d\left(u_{k}, t\right), \\
\frac{1}{2 s} d\left(G u_{k}, G^{2} u_{k}\right) \geq d\left(G u_{k}, t\right) .
\end{gathered}
$$

From (14) and $\left(F_{1}\right)$, we have

$$
d\left(G u_{k}, G^{2} u_{k}\right)<d\left(u_{k}, G u_{k}\right)
$$

It follows from (48) and (49) that

$$
\begin{aligned}
d\left(u_{k}, G u_{k}\right) & \leq s d\left(u_{k}, t\right)+s d\left(t, G u_{k}\right) \\
& \leq \frac{1}{2} d\left(u_{k}, G u_{k}\right)+\frac{1}{2} d\left(G u_{k}, G^{2} u_{k}\right) \\
& <\frac{1}{2} d\left(u_{k}, G u_{k}\right)+\frac{1}{2} d\left(u_{k}, G u_{k}\right)=d\left(u_{k}, G u_{k}\right) .
\end{aligned}
$$

This is a contradiction. Hence, (46) holds. Since $G$ is generalized $G_{\perp}$-Suzuki contraction, (46) yields that, for every $n \in \mathbb{N}$, either

$$
\begin{aligned}
\tau+F\left(d\left(G u_{n}, G t\right)\right) \leq & a F\left(d\left(u_{n}, t\right)\right)+b F\left(d\left(u_{n}, G u_{n}\right)\right) \\
& +c F(d(t, G t)), \\
\tau+F\left(d\left(G^{2} u_{n}, G t\right)\right) \leq & a F\left(d\left(G u_{n}, t\right)\right)+b F\left(d\left(G u_{n}, G^{2} u_{n}\right)\right) \\
& +c F(d(t, G t)),
\end{aligned}
$$

holds. On account of $\left(F_{2}\right)$, the limits in (29) and (45) imply that

$$
\lim _{n \rightarrow \infty} F\left(d\left(u_{n}, t\right)=-\infty, \lim _{n \rightarrow \infty} F\left(d\left(u_{n}, G u_{n}\right)=-\infty\right.\right.
$$

Thus, letting $n \longrightarrow \infty$ in (51), we conclude that

$$
\lim _{n \rightarrow \infty} F\left(d\left(G u_{n}, G t\right)=-\infty\right.
$$

Again by $\left(F_{2}\right)$, we observe that

$$
\lim _{n \rightarrow \infty} F\left(d\left(G u_{n}, G t\right)=0 .\right.
$$

By regarding the triangle inequality with (8), we derive that

$$
\begin{aligned}
d(t, G t) & \leq s\left[d\left(t, G u_{n}\right)+d\left(G u_{n}, G t\right)\right] \\
& =s d\left(t, u_{n+1}\right)+s d\left(G u_{n}, G t\right) .
\end{aligned}
$$

By letting $n \longrightarrow \infty$ in the inequality above together with the limits in (45) and (55), we conclude that $d(t, G t)=0$. Thus, $t$ is a fixed point of $G$; that is $t=G t$. Let us analyze the second case (51). Regarding (12), we have

$$
\begin{aligned}
F( & d\left(G^{2} u_{n}, G t\right) \\
< & \tau+F\left(d\left(G^{2} u_{n}, G t\right) \leq a F\left(d\left(G u_{n}, t\right)\right)\right. \\
& +b F\left(d\left(G u_{n}, G^{2} u_{n}\right)\right)+c F(d(t, G t)) \\
= & a F\left(d\left(u_{n+1}, t\right)\right)+b F\left(d\left(u_{n+1}, G u_{n+1}\right)\right)+c F(d(t, G t)) .
\end{aligned}
$$

As it was discussed above, from (29), (45), and $\left(F_{2}\right)$, we conclude that

$$
\lim _{n \rightarrow \infty} F\left(d\left(G^{2} u_{n}, G t\right)=-\infty\right.
$$


From $\left(F_{2}\right)$ equivalently, we get

$$
\lim _{n \rightarrow \infty} F\left(d\left(G^{2} u_{n}, G t\right)=0\right.
$$
that

Again by the triangle inequality together with (8), we find

$$
\begin{aligned}
d(t, G t) & \leq s\left[d\left(t, G^{2} u_{n}\right)+d\left(G^{2} u_{n}, G t\right)\right] \\
& =s d\left(t, u_{n+2}\right)+s d\left(G^{2} u_{n}, G t\right) .
\end{aligned}
$$

By letting $n \longrightarrow \infty$ in the inequality above together with the limits in (45) and (59), we obtain that $d(t, G t)=0$. Thus, $t$ is a fixed point of $G$. Let $h, g \in W$ be two fixed points of $G$ and suppose that $G^{n} h=h \neq g=G^{n} g$ for all $n \in \mathbb{N}$. By choice of $u_{0}$, we obtain

$$
\left(u_{0} \perp h \text { and } u_{0} \perp g\right) \text { or }\left(h \perp u_{0} \text { and } g \perp u_{0}\right) \text {. }
$$

Since $G$ is $\perp$-preserving, we have

$\left(G^{n} u_{0} \perp G^{n} h\right.$ and $\left.G^{n} u_{0} \perp G^{n} g\right)$ or $\left(G^{n} h \perp G^{n} u_{0}\right.$ and $\left.G^{n} g \perp G^{n} u_{0}\right)$,

for all $n \in \mathbb{N}$. Now,

$$
d(h, g)=d\left(G^{n} h, G^{n} g\right) \leq s\left(d\left(G^{n} h, G^{n} u_{0}\right)+d\left(G^{n} u_{0}, G^{n} g\right)\right)
$$

As $n \longrightarrow \infty$, we obtain $d(h, g) \leq 0$. Thus, $h=g$. Hence, $G$ has a unique fixed point in $W$.

Example 15. Let $W=[0,1] \cup\{2,4\}$ and $d: W \times W \longrightarrow 0$, $\infty)$ be a mapping defined by

$$
d(h, g)=|h-g|^{2}
$$

for all $h, g \in W$. Define the binary relation $\perp$ on $W$ by $h \perp g$ if $h g \leq(h \vee g)$, where $h \vee g=h$ or $g$. Then, $(W, d)$ is an $O$ complete $b$-metric space. Define the mapping $G: W \longrightarrow$ $W$ by

$$
G(g)=\left(\begin{array}{ll}
\ln \left(1+\frac{g}{2}\right) & \text { if } g \in[0,1], \\
g-1 & \text { if } g \in\{2,4\}
\end{array}\right.
$$

Let $a=1 / 2, b=1 / 4, c=1 / 4$ and $\tau>0$. Let $h=2, g=4$, then, $h g \leq g$. We have

$$
d(G h, G g)=4, d(h, g)=4, d(h, G h)=1 \text {, and } d(g, G g)=1 \text {. }
$$

Therefore,

$$
\begin{aligned}
\forall u, v \in & W \text { with } u \perp v\left[d(G u, G v)>0, \frac{1}{2 s} d(u, G u)<d(u, v)\right. \\
\Longrightarrow & \tau+d(G h, G g) \geq a F(d(h, g))+b F(d(h, G h)) \\
& +c F(d(g, G g)) .
\end{aligned}
$$

Hence, $G$ is not generalized $F$-Suzuki contraction mapping, and so Theorem 9 in [3] is not applicable. Now, let us consider the mapping $F$ defined by $F(t)=\ln t, t \in(0, \infty)$. Then, $G$ is $G_{\perp}$-Suzuki contraction with $\tau=\ln 4, c \in[0,1)$, and $a, b \in[0,1]$ are real numbers, $a+b+c=1$. First, we observe that

$$
\begin{gathered}
\forall u, v \in W \text { with } u \perp v\left[d(G u, G v)>0, \frac{1}{2 s} d(u, G u)<d(u, v)\right. \\
\Longleftarrow[h, g \in[0,1] \vee h \in[0,1] \text { and } g \in\{2,4\} \\
\quad \vee h \in\{2,4\} \text { and } g \in[0,1]] .
\end{gathered}
$$

Note that if

$$
\begin{aligned}
\forall u, v & \in W \text { with } u \perp v\left[d(G u, G v)>0, \frac{1}{2 s} d(u, G u)<d(u, v)\right. \\
& \Longrightarrow \tau+F(d(G u, G v)) \\
& \leq a F(d(u, v))+b F(d(u, G u))+c F(d(v, G v))]
\end{aligned}
$$

Clearly, $G$ is $\perp$-preserving. Let $h \perp g$. Without loss of generality, we may assume that $h g \leq g$. Then, we consider the following cases:

Case 16. Let $h, g \in[0,1]$, then $d(G h, G g)=\mid \ln (1+(h / 2))-$ $\left.\ln (1+(g / 2))\right|^{2}, \quad d(h, g)=|h-g|^{2}, \quad d(h, G h)=\mid h-\ln (1+$ $(h / 2))\left.\right|^{2}$, and $d(g, G g)=|g-\ln (1+(g / 2))|^{2}$. It is clear that (69) is satisfied.

Case 17. Let $h \in[0,1]$ and $g \in\{2,4\}$, then $d(G h, G g)=$ $|\ln (1+(h / 2))-g+1|^{2}, d(h, g)=|h-g|^{2}, \quad d(h, G h)=\mid h-$ $\left.\ln (1+(h / 2))\right|^{2}$, and $d(g, G g)=1$. It is clear that (69) is satisfied.

Case 18. Let $h \in\{2,4\}$ and $g \in[0,1]$, then $d(G h, G g)=$ $|h-1-\ln (1+(g / 2))|^{2}, d(h, g)=|h-g|^{2}, d(h, G h)=1$, and $d(g, G g)=|g-\ln (1+(g / 2))|^{2}$. It is clear that (69) is satisfied. Hence, all the hypothesis of Theorem 14 are satisfied, note that $G$ has a unique fixed point $g=0$.

Theorem 19. Let $(W, \perp, d)$ be an $O$-complete orthogonal bmetric space with constant $s \geq 1$ and an orthogonal element 
$u_{0}$ and a mapping $G: W \longrightarrow W$. Suppose that there exist $F \in \mathfrak{I}$ and $\tau>0$ such that the following conditions hold:

(i) $G$ is $\perp$-preserving

(ii) $G$ is a $G_{\perp}$-Suzuki contraction mapping

Then, $G$ has a unique fixed point $t \in W$, and for every $u \in W$, the sequence $\left\{G^{n} u\right\}$ converges to $t$.

Proof. By taking $a=1$ and $b=c=0$ in Theorem 14 , the proof is complete.

Example 20. Let $W=[0,1] \cup\{4,6\}$ and $d: W \times W \longrightarrow 0$, $\infty)$ be a mapping defined by

$$
d(h, g)=|h-g|^{2}
$$

for all $h, g \in W$. Define the binary relation $\perp$ on $W$ by $h \perp g$ if $h g \leq(h \vee g)$, where $h \vee g=h$ or $g$. Then, $(W, d)$ is an $O$-complete $b$-metric space. Define the mapping $G: W \longrightarrow$ $W$ by

$$
G(g)=\left(\begin{array}{ll}
\ln \left(1+\frac{g}{3}\right) & \text { if } g \in[0,1], \\
g-1 & \text { if } g \in\{4,6\}
\end{array}\right.
$$

Let $h=2, g=4$, then $h g \leq g$. Since $d(G 4, G 6)=4=d(4,6)$, then for all $F \in \mathfrak{I}$ and $\tau>0$, we have

$$
\tau+F(d(G 4, G 6))>F(d(4,6))
$$

Therefore, $G$ is not F-Suzuki contraction, and so Theorem 11 in [3] is not applicable. Now, let us consider the mapping $F$ defined by $F(t)=\ln t, t \in(0, \infty)$. Then, $G$ is $G_{\perp}$-Suzuki contraction with $\tau=\ln 4$. First, we observe that

$$
\begin{gathered}
\forall u, v \in W \text { with } u \perp v\left[d(G u, G v)>0, \frac{1}{2 s} d(u, G u)<d(u, v)\right. \\
\Longleftarrow[h, g \in[0,1] \vee h \in[0,1] \text { and } g \in\{4,6\} \\
\quad \vee h \in\{4,6\} \text { and } g \in[0,1]] .
\end{gathered}
$$

Note that if

$$
\begin{aligned}
\forall u, v & \in W \text { with } u \perp v\left[d(G u, G v)>0, \frac{1}{2 s} d(u, G u)<d(u, v)\right. \\
& \Longrightarrow \tau+F(d(G u, G v)) \leq F(d(u, v))
\end{aligned}
$$

Clearly, $G$ is $\perp$-preserving. Let $h \perp g$. Without loss of generality, we may assume that $h g \leq g$. Then, we consider the following cases:

Case 21. Let $h, g \in[0,1]$, then $d(G h, G g)=\mid \ln (1+(h / 3))-$ $\left.\ln (1+(g / 3))\right|^{2}, \quad d(h, g)=|h-g|^{2}, \quad d(h, G h)=\mid h-\ln (1+$ $(h / 3))\left.\right|^{2}$, and $d(g, G g)=|g-\ln (1+(g / 3))|^{2}$. It is clear that (74) is satisfied.
Case 22. Let $h \in[0,1]$ and $g \in\{4,6\}$, then $d(G h, G g)=$ $|\ln (1+(h / 3))-g+1|^{2}, \quad d(h, g)=|h-g|^{2}, \quad d(h, G h)=\mid h-$ $\left.\ln (1+(h / 3))\right|^{2}$, and $d(g, G g)=1$. It is clear that (74) is satisfied.

Case 23. Let $h \in\{4,6\}$ and $g \in[0,1]$, then $d(G h, G g)=$ $|h-1-\ln (1+(g / 3))|^{2}, d(h, g)=|h-g|^{2}, d(h, G h)=1$, and $d(g, G g)=|g-\ln (1+(g / 3))|^{2}$. It is clear that (74) is satisfied. Hence, all the hypothesis of Theorem 19 are satisfied, note that $G$ has a unique fixed point $g=0$.

Corollary 24. Let $G$ be a self mapping on an $O$-complete metric space $(W, \perp, d)$. Assume that there exist $F \in \mathfrak{I}$ and $\tau>0$ such that the following condition hold:

(i) $G$ is $\perp$-preserving

(ii) $\forall u, v \in W$ with $u \perp v[d(G u, G v)>0,1 / 2 d(u, G u)<d$ $(u, v) \Longrightarrow \tau+F(d(G u, G v)) \leq a F(d(u, v))+b F(d(u$, $G u))+c F(d(v, G v))]$

where $c \in[0,1)$ and $a, b \in[0,1]$ are real numbers with $a+$ $b+c=1$.

Then, $G$ has a unique fixed point $t \in W$.

Proof. Since any metric space is a $b$-metric space with constant $s=1$, so from Theorem 14 the proof is complete.

Corollary 25. Let $G$ be a self mapping on an $O$-complete metric space $(W, \perp, d)$. Assume that there exist $F \in \mathfrak{I}$ and $\tau>0$ such that the following condition hold:

(i) $G$ is $\perp$-preserving

(ii) $\forall u, v \in W$ with $u \perp v[d(G u, G v)>0,1 / 2 d(u, G u)<d$ $(u, v) \Longrightarrow \tau+F(d(G u, G v)) \leq F(d(u, v))]$

Then, G has a unique fixed point $t \in W$.

Proof. Since any metric space is a $b$-metric space with constant $s=1$, so by taking $a=1$ and $b=c=0$ from Theorem 14 , the proof is complete.

Proof. By taking $a=1$ and $b=c=0$ in Theorem 14, the proof is complete.

Next, we give the fixed point theorem for orthogonal continuous mapping on an $O$-complete $b$-metric space $(W, \perp, d)$.

Theorem 26. Let $(W, \perp, d)$ be an $O$-complete $b$-metric space with constant $s \geq 1$ and an orthogonal element $u_{0}$ and $a$ mapping $G: W \longrightarrow W$. Suppose that there exist $F \in \mathfrak{I}$ and $\tau>0$ such that the following conditions hold:

(i) $G$ is $\perp$-preserving

(ii) $\forall u, v \in W$ with $u \perp v[d(G u, G v)>0 \Longrightarrow \tau+F(d(G u$, $G v))<a F(d(u, v))+b F(d(u, G u))+c F(d(v, G v))]$

where $c \in[0,1)$ and $a, b \in[0,1]$ are real numbers with $a+b+c=1$. 
(iii) $G$ is $\perp$-continuous

Then, G has a unique fixed point $t \in W$.

Proof. Since $(W, \perp)$ is an $O$-set,

$\exists u_{0} \in W:\left(\forall u \in W, u \perp u_{0}\right)$ or $\left(\forall u \in W, u_{0} \perp u\right)$.

It implies that $u_{0} \perp G u_{0}$ or $G u_{0} \perp u_{0}$. Let

$$
u_{1}:=G u_{0}, u_{2}:=G u_{1}=G^{2} u_{0} \cdots, u_{n+1}:=G u_{n}=G^{n+1} u_{0} \text {, }
$$

for all $n \in \mathbb{N} \cup\{0\}$.

If $u_{n}=u_{n+1}$ for any $n \in \mathbb{N} \cup\{0\}$, then it is clear that $u_{n}$ is a fixed point of $G$. Assume that $u_{n} \neq u_{n+1}$ for all $n \in \mathbb{N} \cup\{0\}$. Therefore, we have $d\left(G u_{n}, G u_{n+1}\right)>0$ for all $n \in \mathbb{N} \cup\{0\}$. As $G$ is $\perp$-preserving, we have

$$
u_{n} \perp u_{n+1} \text { or } u_{n+1} \perp u_{n}
$$

for all $n \in \mathbb{N} \cup\{0\}$. It implies that $\left\{u_{n}\right\}$ is an $O$-sequence. By condition (ii), we have

$$
\left.0<d\left(u_{n}, G u_{n}\right)\right)=d\left(G u_{n-1}, G u_{n}\right) . \forall n \in \mathbb{N} .
$$

So we have

$$
\begin{aligned}
\tau+ & F\left(d\left(G u_{n-1}, G u_{n}\right)\right) \\
& \leq a F\left(d\left(u_{n-1}, u_{n}\right)\right)+b F\left(d\left(u_{n-1}, G u_{n-1}\right)\right)+c F\left(d\left(u_{n}, G u_{n}\right)\right) \\
& =a F\left(d\left(u_{n-1}, u_{n}\right)\right)+b F\left(d\left(u_{n-1}, u_{n}\right)\right)+c F\left(d\left(G u_{n-1}, G u_{n}\right)\right) .
\end{aligned}
$$

Thus,

$$
\tau+(1-c) F\left(d\left(G u_{n-1}, G u_{n}\right)\right) \leq(a+b) F\left(d\left(u_{n-1}, u_{n}\right)\right) .
$$

As $a+b+c=1$, we get

$F\left(d\left(G u_{n-1}, G u_{n}\right)\right) \leq F\left(d\left(u_{n-1}, u_{n}\right)\right)-\frac{\tau}{a+b}<F\left(d\left(u_{n-1}, u_{n}\right)\right)$.

From $\left(F_{1}\right)$, we conclude that

$$
d\left(u_{n}, G u_{n}\right)=d\left(G u_{n-1}, G u_{n}\right)<d\left(u_{n-1}, G u_{n-1}\right), \forall n \in \mathbb{N} .
$$

Thus, $\left\{d\left(u_{n}, G u_{n}\right)\right\}_{n=1}^{\infty}$ is a decreasing sequence of real numbers which is bounded below. Therefore, $\left\{d\left(u_{n}\right.\right.$, $\left.\left.G u_{n}\right)\right\}_{n=1}^{\infty}$ converges and

$$
\lim _{n \rightarrow \infty} d\left(u_{n}, G u_{n}\right)=\delta=\inf \left\{d\left(u_{n}, G u_{n}\right): \forall n \in \mathbb{N}\right\} .
$$

Next, we show that $\delta=0$. Arguing by contradiction, we get $\delta>0$. For every $\varepsilon>0$, there exits $k \in \mathbb{N}$, such that

$$
d\left(u_{k}, G u_{k}\right)<\delta+\varepsilon .
$$

From $\left(F_{1}\right)$, we get

$$
F\left(d\left(u_{k}, G u_{k}\right)\right)<F(\delta+\varepsilon) .
$$

On the other hand (78), we have

$$
\left.0<d\left(u_{k}, G u_{k}\right)\right)=d\left(u_{k-1}, G u_{k}\right)
$$

So we obtain

$$
\begin{aligned}
\tau+ & F\left(d\left(G u_{k-1}, G u_{k}\right)\right) \\
& \leq a F\left(d\left(u_{k-1}, u_{k}\right)\right)+b F\left(d\left(u_{k-1}, G u_{k-1}\right)\right)+c F\left(d\left(u_{k}, G u_{k}\right)\right) \\
& =a F\left(d\left(u_{k-1}, u_{k}\right)\right)+b F\left(d\left(u_{k-1}, u_{k}\right)\right)+c F\left(d\left(G u_{k-1}, G u_{k}\right)\right),
\end{aligned}
$$

and thus

$$
\tau+(1-c) F\left(d\left(G u_{k-1}, G u_{k}\right)\right)<(a+b) F\left(d\left(u_{k-1}, u_{k}\right)\right) .
$$

Since $a+b+c=1$, we obtain

$$
F\left(d\left(G u_{k-1}, G u_{k}\right)\right) \leq F\left(d\left(u_{k-1}, u_{k}\right)\right)-\frac{\tau}{a+b} .
$$

Also from (78), we have $\left.0<d\left(G u_{k}, G u_{k+1}\right)\right)<d\left(G u_{k}\right.$, $\left.G^{2} u_{k}\right)$, and thus, by assumption of theorem, we have

$$
\begin{aligned}
\tau+ & F\left(d\left(G u_{k}, G u_{k+1}\right)\right) \\
& \leq a F\left(d\left(u_{k}, u_{k+1}\right)\right)+b F\left(d\left(u_{k}, G u_{k}\right)\right)+c F\left(d\left(u_{k+1}, G u_{k+1}\right)\right) \\
& =a F\left(d\left(u_{k}, u_{k+1}\right)\right)+b F\left(d\left(u_{k}, u_{k+1}\right)\right)+c F\left(d\left(G u_{k}, G u_{k+1}\right)\right),
\end{aligned}
$$

and therefore,

$$
\tau+(1-c) F\left(d\left(G u_{k}, G u_{k+1}\right)\right) \leq(a+b) F\left(d\left(u_{k}, G u_{k+1}\right)\right) .
$$

Since $a+b+c=1$, we get

$$
F\left(d\left(G u_{k}, G u_{k+1}\right)\right) \leq F\left(d\left(u_{k}, G u_{k+1}\right)\right)-\frac{\tau}{a+b} .
$$


Now by using (85) and continuing similar method as used in (89) and (92), we obtain

$$
\begin{aligned}
F\left(d\left(u_{k+n}, u_{k+n+1}\right)\right) & =F\left(d\left(G u_{k+n-1}, G u_{k+n}\right)\right) \\
& \leq F\left(d\left(u_{k+n-1}, u_{k+n}\right)\right)-\frac{\tau}{a+b} \\
& =F\left(d\left(G u_{k+n-2}, G u_{k+n-1}\right)\right)-\frac{\tau}{a+b} \\
& \leq F\left(d\left(u_{k+n-2}, u_{k+n-1}\right)\right)-\frac{2 \tau}{a+b} \\
& =F\left(d\left(G u_{k+n-3}, G u_{k+n-2}\right)\right)-\frac{2 \tau}{a+b} \\
& \leq F\left(d\left(u_{k+n-3}, u_{k+n-2}\right)\right)-\frac{3 \tau}{a+b} \vdots \\
& \leq F\left(d\left(u_{k+1}, u_{k+2}\right)\right)-\frac{(n-1) \tau}{a+b} \\
& =F\left(d\left(G u_{k}, G u_{k+1}\right)\right)-\frac{(n-1) \tau}{a+b} \\
& \leq F(\delta+\varepsilon)-\frac{n \tau}{a+b} .
\end{aligned}
$$

Letting $n \longrightarrow \infty$, we find that

$$
\lim _{n \rightarrow \infty} F\left(d\left(u_{k+n}, u_{k+n+1}\right)\right)=-\infty
$$

Consequently, from $\left(F_{2}\right)$, we have $\lim _{n \rightarrow \infty} d\left(d\left(u_{k+n}\right.\right.$, $\left.\left.u_{k+n+1}\right)\right)=0$. Thus, there exists $N_{1} \in \mathbb{N}$ such that

$$
d\left(u_{k+n}, u_{k+n+1}\right)<\delta, \forall n \geq N_{1}
$$

and from (76), we get

$$
d\left(u_{k+n}, G u_{k+n}\right)<\delta, \forall n \geq N_{1} .
$$

This is a contradiction with the definition of $\delta$. So $\delta=0$ and from (85) we have

$$
\left.\lim _{n \rightarrow \infty} d\left(u_{n}, G u_{n}\right)\right)=0 .
$$

Next, we claim that

$$
\left.\lim _{n, k \rightarrow \infty} d\left(u_{n}, u_{k}\right)\right)=0
$$

Suppose, otherwise, that there exists $\varepsilon>0$ and sequences $\{i(n)\}_{n=1}^{\infty}$ and $\{j(n)\}_{n=1}^{\infty}$ of natural numbers such that

$i(n)>j(n)>n, d\left(u_{i(n)}, u_{j(n)}\right) \geq \varepsilon, d\left(u_{i(n)-1}, u_{j(n)}\right)<\varepsilon, \forall n \in \mathbb{N}$.
From the triangle inequality, we have

$$
\begin{aligned}
d\left(u_{i(n)}, u_{j(n)}\right) & \leq s\left[d\left(u_{i(n)}, u_{i(n)-1}\right)+d\left(u_{i(n)-1}, u_{j(n)}\right)\right] \\
& \leq s d\left(u_{i(n)}, u_{i(n)-1}\right)+s \mathcal{E} \\
& =s d\left(u_{i(n)-1}, G u_{i(n)-1}\right)+s \varepsilon, \forall n \in \mathbb{N}
\end{aligned}
$$

Due to (97), there exists an $N_{2} \in \mathbb{N}$ such that

$$
d\left(u_{i(n)}, G u_{i(n)}\right)<\varepsilon, \forall n>N_{2}
$$

It implies from (100) and (101) that

$$
d\left(u_{i(n)}, u_{j(n)}\right)<2 s \varepsilon, \forall n>N_{2}
$$

So from $\left(F_{2}\right)$, we obtain

$$
F\left(d\left(u_{i(n)}, u_{j(n)}\right)\right)<F(2 s \varepsilon), \forall n>N_{2}
$$

Also from (99), we can easily get that

$$
\varepsilon \leq d\left(u_{i(n)+1}, u_{j(n)+1}\right)=d\left(G u_{i(n)}, G u_{j(n)}\right), \forall n>N_{2} .
$$

Therefore, from (103) and assumption of theorem, we get

$$
\begin{aligned}
\tau+ & F\left(d\left(G u_{i(n)}, G u_{j(n)}\right)\right) \\
\leq & a F\left(d\left(u_{i(n)}, u_{j(n)}\right)\right)+b F\left(d\left(u_{i(n)}, G u_{i(n)}\right)\right) \\
& +c F\left(d\left(u_{j(n)}, G u_{j(n)}\right)\right) \\
< & a F(2 s \varepsilon)+b F\left(d\left(u_{i(n)}, G u_{i(n)}\right)\right) \\
& +c F\left(d\left(u_{j(n)}, G u_{j(n)}\right)\right), \forall n \in \mathbb{N} .
\end{aligned}
$$

Using (100) and $\left(F_{2}\right)$, we obtain

$$
\lim _{n \rightarrow \infty} F\left(d\left(G u_{i(n)}, G u_{j(n)}\right)\right)=-\infty \text {. }
$$

Thus, from this and $\left(F_{2}\right)$, we get

$\lim _{n \rightarrow \infty} F\left(d\left(G u_{i(n)}, G u_{j(n)}\right)\right)=0 \Leftrightarrow \lim _{n \rightarrow \infty} F\left(d\left(u_{i(n)+1}, u_{j(n)+1}\right)\right)=0$.

A contradiction with relations in (99). Hence, $\lim _{k, n \rightarrow \infty}$ $d\left(u_{n}, u_{k}\right)=0$. On the account of completeness of $(W, d)$, there exists $t \in W$ such that

$$
\lim _{n \rightarrow \infty} d\left(u_{n}, t\right)=0
$$


Since $G$ is $\perp$-continuous, we get

$$
\lim _{n \rightarrow \infty} d\left(G u_{n}, G t\right)=0 .
$$

As $d(t, G t) \leq s\left[d\left(t, u_{n}\right)+d\left(x_{n}, G t\right)\right]$, thus, $d(t, G t)=0$ and so $t$ is a fixed point of $G$.

Let $h, g \in W$ be two fixed points of $G$ and suppose that $G^{n} h=h \neq g=G^{n} g$ for all $n \in \mathbb{N}$. By choice of $u_{0}$, we obtain

$$
\left(u_{0} \perp h \text { and } u_{0} \perp g\right) \text { or }\left(h \perp u_{0} \text { and } g \perp u_{0}\right) \text {. }
$$

Since $G$ is $\perp$-preserving, we have

$$
\left(G^{n} u_{0} \perp G^{n} h \text { and } G^{n} u_{0} \perp G^{n} g\right) \text { or }\left(G^{n} h \perp G^{n} u_{0} \text { and } G^{n} g \perp G^{n} u_{0}\right)
$$

for all $n \in \mathbb{N}$. Now,

$$
d(h, g)=d\left(G^{n} h, G^{n} g\right) \leq s\left(d\left(G^{n} h, G^{n} u_{0}\right)+d\left(G^{n} u_{0}, G^{n} g\right)\right) .
$$

As $n \longrightarrow \infty$, we obtain $d(h, g) \leq 0$. Thus, $h=g$. Hence, $G$ has a unique fixed point in $W$.

Example 27. Let $W=\mathbb{R}$ and $d: W \times W \longrightarrow 0, \infty)$ be a mapping defined by

$$
d(h, g)=\max \{h, g\}^{2}
$$

for all $h, g \in W$. Define the binary relation $\perp$ on $W$ by $h \perp g$ if $h, g \in[3, n+4]$ for some $n \in \mathbb{N}$ or $h=0$. Then, $(W, d)$ is an $O$-complete $b$-metric space. Define the mapping $G: W \longrightarrow W$ by

$$
G(g)=\left(\begin{array}{ll}
0 & \text { if } g=0 \\
g+1 & \text { if } g \in[1,2], \\
g+1 & \text { if } g \in(2, \infty) .
\end{array}\right.
$$

Let $h \in W$ and $\left\{h_{n}\right\}$ be an arbitrary $\perp$-sequence in $W$ such that converges to $h$, then the following cases are satisfied:

Case 28. If $h_{n}=0$ for all $n$, then $h=0$ and $G\left(h_{n}\right)=0=G(h)$.

Case 29. If $h_{n} \neq 0$ for some $n$, then there exists $m \in \mathbb{N}$ such that $h \in[3, m+4]$, and $G\left(h_{n}\right)=G(h)$. Therefore, $G$ is $\perp$-continuous on $W$, but it is not continuous on $W$. Let $a=1 / 2, b=1 / 4$, and $c=1 / 4$. Since $d(G 1, G 2)=9, d(1,2)=4$, $d(1, G 1)=4$, and $d(2, G 2)=9$, then for all $F \in \mathfrak{I}$ and $\tau>0$ we have

$$
\begin{aligned}
& \forall h, g \in W \text { with } h \perp g[d(G h, G g)>0 \\
& \Longrightarrow \\
& \quad+c F(d(g h, G g) \geq a F(d(h, g))+b F(d(h, G h))
\end{aligned}
$$

Therefore, Theorem 16 in [3] is not applicable. Now, let us consider the mapping $F$ defined by $F(t)=\ln t, t \in(0, \infty)$. First, we observe that

$\forall h, g \in W$ with $h \perp g[d(G h, G g)>0 \Leftarrow[h=0$ and $g \in(2, \infty)]$.

\section{Note that if}

$$
\begin{aligned}
& \forall h, g \in W \text { with } h \perp g\left[d(G h, G g)>0, \frac{1}{2 s} d(h, G h)<d(h, g)\right. \\
& \Longrightarrow \tau+F(d(G h, G g)) \leq a F(d(h, g))+b F(d(h, G h)) \\
& \quad+c F(d(g, G g))]
\end{aligned}
$$

Clearly, $G$ is $\perp$-preserving. Let $h \perp g$ and $h=0, g \in(2, \infty)$, $\tau=\ln 2$, then $d(G h, G g)=(g-1)^{2}, d(h, g)=g^{2}, d(h, G h)=$ 0 , and $d(g, G g)=g^{2}$. It is clear that (117) is satisfied.

Hence, all the hypothesis of Theorem 26 are satisfied, note that $G$ has a unique fixed point $g=0$.

Theorem 29. Let $(W, \perp, d)$ be an $O$-complete $b$-metric space with constant $s \geq 1$ and an orthogonal element $u_{0}$ and $a$ mapping $G: W \longrightarrow W$. Suppose that there exist $F \in \mathfrak{I}$ and $\tau>0$ such that the following conditions hold:

(i) $G$ is $\perp$-preserving $\forall u, v \in W$ with $u \perp v[d(G u, G v)>0$ $\Longrightarrow \tau+F(d(G u, G v))<F(d(u, v))]$;

(ii) $G$ is $\perp$-continuous

Then, $G$ has a unique fixed point $t \in W$.

Proof. By taking $a=1$ and $b=c=0$ in Theorem 26, the proof is complete.

Theorem 30. Let $(W, \perp, d)$ be an $O$-complete metric space. Suppose that there exist $F \in \mathfrak{I}$ and $\tau>0$ such that the following conditions hold:

(i) $G$ is $\perp$-preserving

(ii) $\forall u, v \in W$ with $u \perp v[d(G u, G v)>0 \Longrightarrow \tau+F(d(G u$, $G v))<a F(d(u, v))+b F(d(u, G u))+c F(d(v, G v))]$,

where $c \in[0,1)$ and $a, b \in[0,1]$ are real numbers with $a+b+c=1$

(iii) $G$ is $\perp$-continuous

Then, $G$ has a unique fixed point $t \in W$.

Proof. It is sufficient to take $s=1$ in Theorem 26 the proof is complete. 
Theorem 31. Let $(W, \perp, d)$ be an $O$-complete metric space. Suppose that there exist $F \in \mathfrak{I}$ and $\tau>0$ such that the following conditions hold:

(i) $G$ is $\perp$-preserving

(ii) $\forall u, v \in W$ with $u \perp v[d(G u, G v)>0 \Longrightarrow \tau+F(d(G u$, $G v))<F(d(u, v))]$

(iii) $G$ is $\perp$-continuous

Then, G has a unique fixed point $t \in W$.

Proof. Since every metric space is a $b$-metric space with constant $s=1$, from Theorem 29, $G$ has a fixed point $t \in W$. Indeed, if there is another fixed point $s \in W$ of $G$, such that $\forall t, s \in W$ with $t \perp s[d(G t, G s)>0$. Since $\tau>0$, from our assumption of theorem, we obtain

$$
F(d(t, s))=F(d(G t, G s))<\tau+F(d(G t, G s)) \leq a F(d(t, s)) .
$$

This is contradiction. So $G$ has a unique fixed point.

\section{Application to Ordinary Differential Equations}

For any $1 \leq p<\infty$, the space $L^{p}(W, F, \lambda)$ (or $L^{p}(W)$ ) consists of all complex-valued measurable $\beta$ on the underlying space $W$ satisfying

$$
\int_{W}|\beta(w)|^{p} d \lambda(w) .
$$

Here $F$ is the $\sigma$-algebra of measurable sets and $\lambda$ is the measure.

When $p=1$, the space $L^{1}(W)$ consists of all integrable functions $\beta$ on $W$. We define the $L^{1}$-norm of $\beta$ by

$$
\|\beta\|_{1}=\int_{W}|\beta(w)| d \lambda(w) .
$$

Now using Theorem 19, we prove the existence of a solution of the differential equation:

$$
\begin{aligned}
& j^{\prime}(t)=G(t, j(t)), \text { a.e. } t \in I:=[0, T], \\
& j(0)=c, c \geq 1,
\end{aligned}
$$

where $G: I \times \mathbb{R} \longrightarrow \mathbb{R}$ is an integrable function satisfying the conditions:

(i) $G(s, y) \geq 0$ for all $y \geq 0$ and $s \in I$ (ii) for each $l, m \in L^{1}(I)$ with $l(s) m(s) \geq l(s)$ or $l(s) m(s)$ $\geq m(s)$ for all $s \in I$, there exist $\gamma \in L^{1}(I)$ and $\tau>0$ such that

$$
\begin{aligned}
|G(s, l(s))-G(s, m(s))| & \leq \frac{\gamma(s)}{1+\tau \gamma(s)}|l(s)-m(s)|^{2} \\
|l(s)-m(s)|^{2} & \leq \gamma(s) e^{2 B(s)}
\end{aligned}
$$

for all $s \in I$, where $B(s):=\int_{0}^{s}|\gamma(r)| d r$.

Theorem 32. Consider the differential Eq. ((122)). If (i) and (ii) are satisfied, then the differential Eq. ((122)) has a unique positive solution.

Proof. Let $W=\left\{w \in L^{1}(W): w(t)>0\right.$ for all $\left.t \in I\right\}$. Define the orthogonality relation $\perp$ on $V$ by

$$
l \perp m \Leftarrow l(t) m(t) \geq l(t) \text { or } l(t) m(t) \geq m(t) \text { for all } t \in I .
$$

As $B(t)=\int_{0}^{t}|\gamma(s)| d s$, we have $B^{\prime}(t)=|\gamma(t)|$ for almost everywhere $t \in I$. Now define a mapping $d: W \times W \longrightarrow 0$,

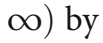

$$
d(l, m)=\|l-m\|_{B}=\sup _{t \in I} e^{-2 B(t)}|l(t)-m(t)|^{2},
$$

for all $l, m \in W$. Therefore, $(W, d)$ is a complete $b$-metric space. Define a mapping $Q: W \longrightarrow W$ by

$$
(Q l)(t)=c+\int_{0}^{t} G(s, l(s)) d s .
$$

Next, we show that $Q$ is $\perp$-preserving. For each $l, m \in W$ with $l \perp m$ and $t \in I$, we have

$$
(Q l)(t)=c+\int_{0}^{t} G(s, l(s)) d s \geq 1 .
$$

It follows that $[(Q l)(t)][(Q m)(t)] \geq(Q m)(t)$ and so $(Q l)(t) \perp(Q m)(t)$. Then, $Q$ is $\perp$-preserving.

Now, we claim that $Q$ is an $G_{\perp}$-Suzuki contraction. Let $l, m \in W$ with $l \perp m$. Suppose that $Q(l) \neq Q(m)$. For each $s \in I$, we have

$$
|l(s)-m(s)|^{2} \leq \gamma(s) e^{2 B(s)}
$$

and so

$$
d(l, m)=\sup _{s \in I} e^{-2 B(s)}|l(s)-m(s)|^{2} \leq \gamma(s) .
$$

Therefore,

$$
1+\tau d(l, m) \leq 1+\tau \gamma(s)
$$


From (ii), for each $t \in I$, we get

$$
\begin{aligned}
|(Q l)(t)-(Q m)(t)| & \leq \int_{0}^{t}|G(s, l(s))-G(s, m(s))| d s \\
& \leq \int_{0}^{t} \frac{|\gamma(s)|}{1+\tau \gamma(s)}|l(s)-m(s)|^{2} d s \\
& \leq \int_{0}^{t} \frac{|\gamma(s)|}{1+\tau d(l, m)}|l(s)-m(s)|^{2} e^{-2 B(s)} e^{2 B(s)} d s \\
& \leq \frac{d(l, m)}{1+\tau d(l, m)} \int_{0}^{t}|\gamma(s)| e^{2 B(s)} d s \\
& \leq \frac{d(l, m)}{1+\tau d(l, m)}\left(e^{2 B(t)}-1\right),
\end{aligned}
$$

and so

$$
\begin{aligned}
e^{-2 B(t)}|(Q l)(t)-(Q m)(t)| & \leq e^{-2 B(t)}\left(e^{2 B(t)}-1\right) \frac{d(l, m)}{1+\tau d(l, m)} \\
& =\left(1-e^{-2 B(t)}\right) \frac{d(l, m)}{1+\tau d(l, m)} \\
& \leq\left(1-e^{-\|\beta\|_{1}}\right) \frac{d(l, m)}{1+\tau d(l, m)} .
\end{aligned}
$$

Thus,

$$
\begin{aligned}
d(Q l, Q m) & \leq \frac{d(l, m)}{1+\tau d(l, m)} \frac{1+\tau d(l, m)}{d(l, m)} \\
& \leq \frac{1}{d(Q l, Q m)} \tau+\frac{1}{d(l, m)} \\
& \leq \frac{1}{d(Q l, Q m)} \tau+\frac{1}{d(l, m)} \\
& \leq \frac{1}{d(Q l, Q m)} \tau-\frac{1}{d(Q l, Q m)} \\
& \leq-\frac{1}{d(l, m)} .
\end{aligned}
$$

Taking a function $F: \mathbb{R}^{+} \longrightarrow \mathbb{R}$ by $F(b)=-1 / b$ for all $b>0$, it implies that $Q$ is an $G_{\perp}$-Suzuki contraction with $a=1, b=0$ and $c=0$. Using Theorem 19, $Q$ has a unique fixed point. Hence, the differential Eq. (122) has a unique positive solution.

\section{Data Availability}

No data was used to support this study.

\section{Conflicts of Interest}

The authors declare that they have no conflict of interest.

\section{References}

[1] I. A. Bakhtin, "The contraction mapping principle in quasimetric spaces," Functional Analysis, vol. 30, pp. 26-37, 1989.
[2] S. Czerwik, "Contraction mapping b-metric spaces," Acta Mathematica et Informatica Universitatis Ostraviensis, pp. 511, 1993.

[3] H. Alsulami, E. Karapinar, and H. Piri, "Fixed points of generalised F-Suzuki type contraction in complete $b$-metric space," Discrete Dynamics in Nature and Society, vol. 2015, Article ID 969726, 8 pages, 2015.

[4] M. E. Gordji, M. Ramezani, M. De La Sen, and Y. J. Cho, "On orthogonal sets and Banach fixed point theorem," Fixed Point Theory, vol. 18, no. 2, pp. 569-578, 2017.

[5] M. Eshaghi Gordji and H. Habibi, "Fixed point theory in generalized orthogonal metric space," Journal of Linear and Topological Algebra, vol. 6, no. 3, pp. 251-260, 2017.

[6] K. Sawangsup, W. Sintunavarat, and Y. J. Cho, "Fixed point theorems for orthogonal $\mathrm{F}$-contraction mappings on $\mathrm{O}$-complete metric spaces," Journal of Fixed Point Theory and Applications, vol. 22, no. 1, p. 10, 2020.

[7] M. Eshaghi and H. Habibi, "Fixed point theory in $\varepsilon$-connected orthogonal metric space," Shand Communications in Mathematical Analysi, vol. 16, no. 1, pp. 35-46, 2019.

[8] N. B. Gungor and D. Turkoglu, "Fixed point theorems on orthogonal metric spaces via altering distance functions," AIP Conference Proceedings, vol. 2183, article 040011, 2019.

[9] O. Yamaod and W. Sintunavarat, "On new orthogonal contractions in b-metric spaces," International J. Pure Mathematics, vol. 5, 2018.

[10] K. Sawangsup and W. Sintunavarat, "Fixed point results for orthogonal $Z$-contraction mappings in $O$-complete metric space," International Journal of Applied Physics and Mathematics, vol. 10, no. 1, 2020.

[11] T. Senapati, L. K. Dey, B. Damjanović, and A. Chanda, "New fixed point results in orthogonal metric spaces with an application," Kragujevac Journal of Mathematics, vol. 42, no. 4, pp. 505-516, 2018.

[12] D. Wardowski, "Fixed points of a new type of contractive mappings in complete metric spaces," Fixed Point Theory and Applications, vol. 2012, no. 1, 2012. 